\title{
Uncontrolled interventions during pandemics: a missed learning opportunity?
}

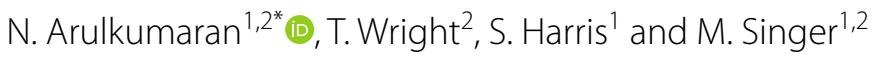

๑ 2020 Springer-Verlag GmbH Germany, part of Springer Nature

\section{Dear Editor,}

Since the start of the COVID-19 pandemic, few randomised clinical trials (RCT) have been published and only one, at the moment available in medrxiv, which shows promise in critically ill patients [1]. With limited conclusive data based on our conventional highest metric for trial efficacy, the RCT, medical professionals feel uneasy, standing back without the option of specific treatment as further lives are lost. With incompletely evaluated data published as preprints or in the media, expectations from patients and relatives on clinicians to prescribe inadequately evaluated therapies can be overwhelming.

In the absence of therapies of proven benefit, therapeutic agents for COVID-19 should be considered within the context of an RCT. However, setting up well-conducted RCTs during an international pandemic is challenging, as reflected by the current paucity of published trials to date. In contrast, several therapeutic interventions, including steroids and monoclonal antibodies, have been used in the absence of a control group, either as compassionate use or due to a lack of equipoise.

These published data are likely to represent the tip of a large iceberg, with a number of clinicians feeling inclined to administer unproven therapies without rigorous analysis or publication of their own data. Inconclusive but promising data may encourage clinicians to use treatments indiscriminately, without any means of accurately evaluating outcomes. The incongruence between international guideline recommendations and clinical

\footnotetext{
*Correspondence: nisharulkumaran@doctors.org.uk

${ }^{1}$ Bloomsbury Institute of Intensive Care Medicine, University College London, Cruciform Building, Gower St, London WC1E 6BT, UK

Full author information is available at the end of the article
}

practice reflects physician unease in not trying any treatment at all, despite the risk of futility or even harm. Such decisions need to be made within the appropriate ethical and legal framework to ensure learning for the benefit of future patients.

Off-label drug use on compassionate grounds also results in administration of interventions in a non-systematic manner, based on the patient's best interests where death is imminent or no alternative therapy exists $[2,3]$. In contrast to research, the primary focus of compassionate use of drugs is improving the outcome of an individual patient, but not to generate scientific data. However, data arising from compassionate prescribing may provide useful information on the efficacy of experimental treatments in accordance with the 'monitored emergency use of unregistered and experimental interventions' (MEURI) guidelines [2, 3]. Such principles are encompassed within the Declaration of Helsinki [4].

Although Meuri guidelines provide a framework to objectively analyse our own practice where uncertainty exists, the degree of learning is limited. This does not preclude learning altogether. As an example, the mechanistic insights into a novel therapy may be evaluated in clinical practice before proceeding to an RCT.

Data gleaned from the compassionate use of therapies is of limited use due to lack of a control group and small numbers of patients. It may not be possible to recognise associated benefit or harm. Death will be ascribed to the underlying disease, whereas improvements will be attributed to the treatment. Additionally, compassionate prescribing decisions are made individually and without formal structure, with high risk of bias and confounding. Due to the quasi-experimental nature of compassionate prescribing, the risk of conflict of interest between

\section{Springer}


vulnerable patients and researchers is not insignificant. This cannot be effectively managed without a regulatory independent of the prescriber.

The ability to learn during pandemics is difficult, but requires a systemic approach with a culture of open reporting. Clinicians ought to take every opportunity to contribute to RCTs. As an example, the Recovery study has provided a pragmatic platform in which patients can be enrolled and useful clinical data established (www. Recoverytrial.net). Additionally, large clinical trials in closely related diseases may be extended to recruit patients during a pandemic. The REMAP-CAP study, originally designed to study treatments for pneumonia, has been rapidly 'repurposed' to investigate therapies in COVID-19. However, where it is not possible to enrol in RCTs, national and international registries should facilitate the collection of observational data on a large scale.

Neither the use of off-label drugs on compassionate grounds, nor prescribing drugs with unknown risk-benefit profiles, should divert attention from the provision of end of life care where death is imminent, divert treatment away from others who are more likely to benefit, or prevent enrolling patients into an RCT. However, under such circumstances, clinicians are obliged to take every opportunity to learn for the sake of our future patients.

\section{Compliance with ethical standards}

Conflict of interest

On behalf of all authors, the corresponding author states that there is no conflict of interest.

\section{Publisher's Note}

Springer Nature remains neutral with regard to jurisdictional claims in published maps and institutional affiliations.

Accepted: 10 July 2020

Published online: 23 July 2020

References

1. RECOVERY Collaborative Group (2020) Effect of Dexamethasone in Hospitalized Patients with COVID-19 - Preliminary report. https://www.medrx iv.org/content/10.1101/2020.06.22.20137273v1. Accessed 26 June 2020

2. WHO (2016) Guidance for managing ethical issues in infectious disease outbreaks. https://www.who.int/ethics/publications/infectious-disea se-outbreaks/en/. Accessed 26 June 2020

3. $\mathrm{WHO}$ (2020) Clinical management of severe acute respiratory infection when novel coronavirus (2019-nCoV) infection is suspected. https:// www.who.int/publications/i/item/clinical-management-of-covid-19. Accessed 26 June 2020

4. WMA (2013) Declaration of Helsinki - Ethical principles for medical research involving human subjects. https://www.wma.net/policies-post/ wma-declaration-of-helsinki-ethical-principles-for-medical-research-invol ving-human-subjects/. Accessed 26 June 2020
Author details

1 Bloomsbury Institute of Intensive Care Medicine, University College London, Cruciform Building, Gower St, London WC1E 6BT, UK. ${ }^{2}$ Critical Care Unit, University College London Hospital, London, UK. 